\title{
Research on Web Service Composition Based on QoS Constraint
}

\author{
Liangda Yan \\ Zhejiang Business Technology Institute, Ningbo, 315012, China \\ email:yanliangda00@sina.com
}

Keywords: Web Service; QoS; Composition

\begin{abstract}
Recently, there has been a growing fashion for businesses to outsource parts of their processes, so as to emphasis more on their core activities. In addition, Web operators often need to compose different services to achieve a more complex task that cannot be fulfilled by an individual service. Web services provide the means for such seamless integration of business processes across organizational boundaries. How to integrate the existent Web services to form a newly value-added and complex service to meet the requirement of different users is a popular research area. This paper discusses the composition of Web Services using QoS. The idea is based on the assumption that for each task in a workflow a set of alternative Web Services with similar functionality is available and that these Web Services have different QoS parameters and costs. Web Service selection with QoS global optimal is transformed into a multi-objective services composition optimization with QoS constraints.
\end{abstract}

\section{Introduction}

Web services are widely applied in various fields to make the Internet a qualitative leap, not only for different departments communication and between different areas of information activities provides a basic platform, and at the same time, the departments and enterprises competition, departments and enterprises to want to in the fierce competition to survive and continue to make progress, you have to make a fast and flexible response to customer needs and changes in the external competition. How to better save previous investments, make full use of existing resources, integrate meets the needs of users, effective value-added service, has become the various departments, enterprises urgently need to solve the problem. In order to disperse and simplify the application logic and improve web services can be reusable, single web service cannot too complex, however in real life applications are generally more complex, such as a travel service usually composed of ticket booking, hotel services and attractions service such as and. When a single web service offers limited function meet the user's needs, you need to will a simple function of the service combination, function more powerful value-added services, to meet the higher demand of users. Therefore, academic and industrial circles, hope can through a combination of existing web services to create new services, both to protect the previous investment of various departments and enterprises, and the integration of a new service function, brings hope for the development of departments and enterprises, the real potential of such web services to play out. Therefore, Web service composition has become one of the important technologies to solve the problems of B2B application in the Web task, and it has become an important research content in industry and academia.

Along with the gradual expansion of the scope of the development of web service technology and its application, more and more enterprises and organizations in succession the continuous will package the business processes into standard web services to publish to the Internet, resulting in the number of services on the Internet increase exponentially. These released by a web service provider web service, inevitably appear lots with the same function but different QoS attributes of the service, the service requester to from quickly found to meet their specific needs and QoS constrained web services. However, the growing number of Web services has tens of thousands of different combination schemes can be combined with the same function but different QoS properties of service, from the scheme to select the optimal scheme is a key problem must be solved in the web 
service composition, otherwise it will affect the further promotion of the service composition applications. Because users of services combination of QoS constraints will be building the combination process of global limits, in support of QoS constrained service portfolio, with global QoS constrained service portfolio accounted for a considerable proportion of; also, due to the different users are concerned about the field and QoS attributes, with the needs of users, also follow different and with service application of the depth and scope of the field continues to expand, and service related QoS attributes and user demand is changing and expanding. Therefore, only according to the change of the user needs to choose a combination of different services, it is possible to maximize the depth and breadth of the application of Web services.

Therefore, according to the web service development present situation and the current situation of the research and application, to the service provider and the service requester's QoS is described. Based on the discovery of Web services, meet the matching user needs the best service, and web service composition according to the user the QoS constraint is a very academic value and practical significance.

\section{QoS constrained portfolio planning framework}

Figure 1 shows the proposed QoS oriented portfolio planning architecture structure, the architecture supports in a user-friendly way to automatically generate QoS oriented web service composition planning, the structure contains a combination of agent and an execution plan optimizer which perform planning optimizer used to implement the algorithm combination.

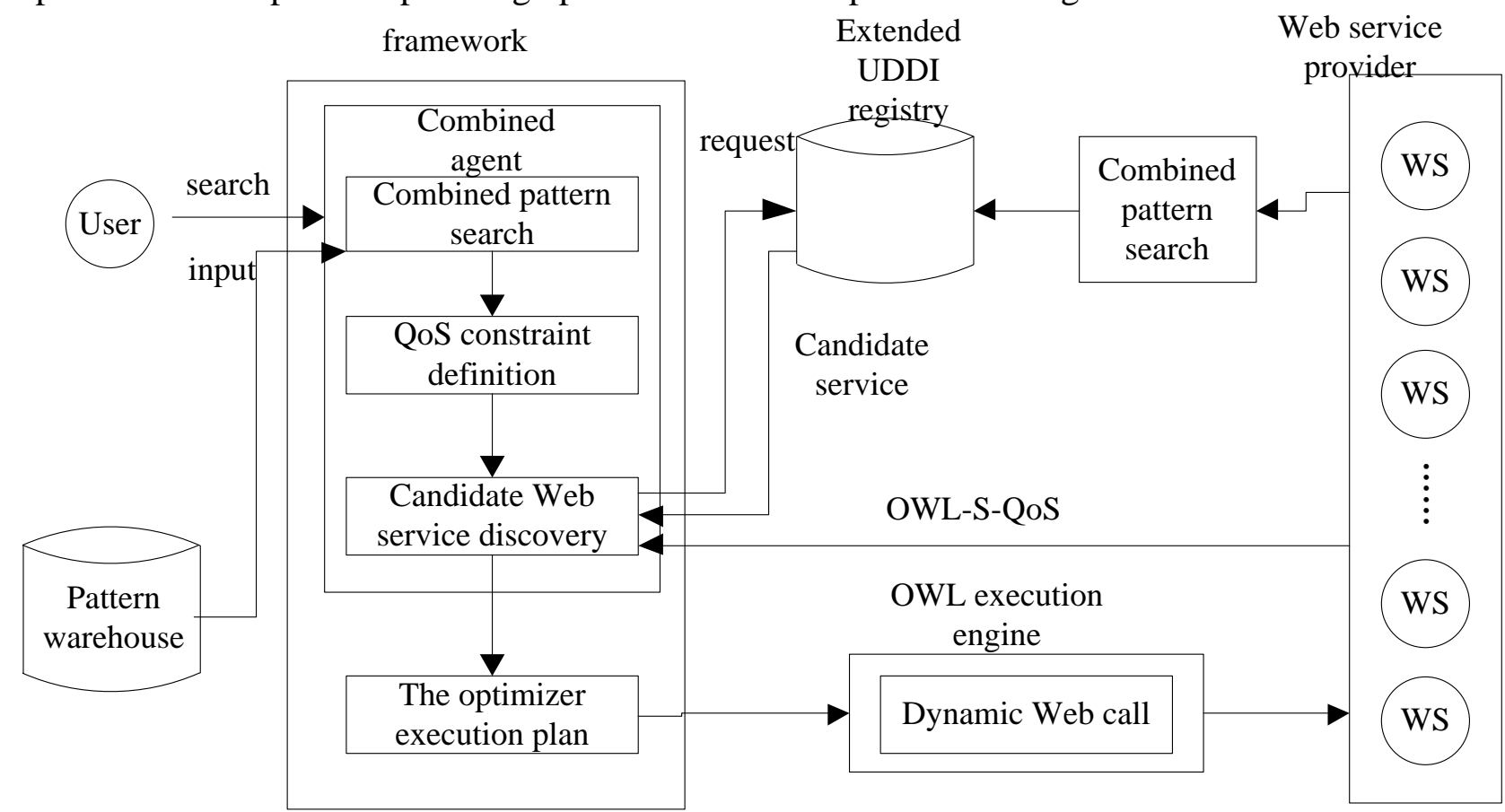

Figure 1 Web service composition planning framework based on QoS

The implementation steps of the architecture are as follows:

By combination of agents to help users find the combination mode, the user through the browsing service category or keyword search in pattern warehouse storage combined mode, then combination agent according to the user specified input weights six QoS attributes of allowed values.

In the selected combination model, the combination model is specified in detail, and the combination agent finds the candidate Web service through the extended UDDI, and retrieves the OWL-S-QoS file from the Web service provider.

In the OWL-S-QoS document on the QoS values are described in detail, the implementation of planning optimizer operation combination algorithm to generate to meet the constraints of the combinatorial planning. If the combination of planning has been recognized by the user, then the 
next only through the implementation of the engine to perform and monitor the combination plan.

\section{Web service composition algorithm based on QoS constraint}

Support QoS constrained web service composition algorithm adopts a combination mode, six QoS can be allowed values and the weights as input and produce a meet the QoS requirement of the user portfolio planning as the output. A Web service can only be located in an atomic process, corresponding to the combination model, the decision value of the collection is a combination of planning.

The main body of the algorithm is based on the tabu search technique. Suppose that a combination of planning by the number of decision variables modification of limited created a better adjacency planning, if this operation does not exist in the tabu list, then the modified operation records in the list, or try another operation, until the operation is not in the list, the adjacency planning generation mechanism will be continued operation until the target function value was reduced to 0 so far. In the generation of adjacency planning, the algorithm will prevent the use of the tabu list of operations, in order to prevent back to the previous visit to the planning. From the optimization point of view, the tabu list has the advantage of avoiding the local optimum.

Table 1 algorithm based on tabu search and simulated annealing algorithm

begin

1. S=search_initial_plan() / * Starting with the initial planning $\mathrm{S} *$ /

2. If $\mathrm{f}(\mathrm{S})=0$ Then $S^{*}=\mathrm{S}$ and terminate

3. For a fixed number of iterations Do

4. $\quad S^{\prime}=$ Generate_neighbor_plan(S) / * Create an adjacency plan */

5. If $\mathrm{f}(\mathrm{s})=\mathrm{O}$ Then $S^{*}=S^{\prime}$ and terminate

6. If $\exp \left\{\left(\mathrm{f}(\mathrm{S})-\mathrm{f}\left(S^{\prime}\right) *\right.\right.$ Iteration $\}>$ random $(0,1)$ Then

7. $\quad$ Accept the move and update current plan as $\mathrm{S}=S^{\prime}$

8. Else

9. $\quad$ Reject the move

10. End If

11. Increase iteration number by one

12. End For

13. $S^{*}=\mathrm{S}$ and return $S^{*}$

End

\section{QoS computing model of composite service}

Generally speaking, portfolio planning have parallel, serial, and parallel and XOR implementation dependent model, table 2 Formula polymerization of component web services QoS attribute values, in accordance with the characteristics of these properties, the serial and parallel mode uses addition and multiplication operators, because each component services are by two executable models is connected, the exception is and parallel mode attribute execution time using the max operator. For XOR parallel mode, the XOR attribute is considered as the worst case for the component services, so the QoS attribute is considered to be the worst case.

The aggregation algorithm is very simple, using the formula in Table 2, to create a virtual service by aggregating the QoS attribute values of the component services connected by the serial mode. All of the serial mode of the aggregation process can be performed. We aggregate the next service that is connected to the AND parallel and XOR parallel mode, and create the corresponding virtual 
service. The aggregation step is always iterated until a virtual service is left.

Table 2 aggregation methods for QoS properties

\begin{tabular}{|l|l|l|l|}
\hline QoS property & serial & \multicolumn{1}{|c|}{ AND parallel } & XOR paralle \\
\hline $\begin{array}{l}\text { Implementation } \\
\text { costs }\end{array}$ & $\sum_{i=m}^{m+n} c\left(s^{i}\right)$ & $\sum_{i=m}^{m+n} c\left(s^{i}\right)$ & $\max \left\{c\left(s_{m}\right), c\left(s_{m+1}\right) \ldots c\left(s_{m+n}\right)\right\}$ \\
\hline Execution time & $\sum_{i=m}^{m+n} t\left(s^{i}\right)$ & $\max \left\{t\left(s_{m}\right), t\left(s_{m+1}\right) \ldots t\left(s_{m+n}\right)\right\}$ & $\max \left\{t\left(s_{m}\right), t\left(s_{m+1}\right) \ldots t\left(s_{m+n}\right)\right\}$ \\
\hline Success rate & $\prod_{i=m}^{m+n} \operatorname{suc}\left(s_{i}\right)$ & $\prod_{i=m}^{m+n} \operatorname{suc}\left(s_{i}\right)$ & $\min \left\{\operatorname{suc}\left(s_{m}\right), \operatorname{suc}\left(s_{m+1}\right) \ldots \operatorname{suc}\left(s_{m+n}\right)\right\}$ \\
\hline Usability & $\prod_{i=m}^{m+n} a v\left(s_{i}\right)$ & $\prod_{i=m}^{m+n} a v\left(s_{i}\right)$ & $\min \left\{\operatorname{av}\left(s_{m}\right), a v\left(s_{m+1}\right) \ldots a v\left(s_{m+n}\right)\right\}$ \\
\hline Reputation & $\frac{1}{n+1} \prod_{i=m}^{m+n} r e p\left(s_{i}\right)$ & $\frac{1}{n+1} \prod_{i=m}^{m+n} r e p\left(s_{i}\right)$ & $\min \left\{r e p\left(s_{m}\right), \operatorname{rep}\left(s_{m+1}\right) \ldots r e p\left(s_{m+n}\right)\right\}$ \\
\hline Frequency & $\frac{1}{n+1} \prod_{i=m}^{m+n} f\left(s_{i}\right)$ & $\frac{1}{n+1} \prod_{i=m}^{m+n} f\left(s_{i}\right)$ & $\min \left\{f\left(s_{m}\right), f\left(s_{m+1}\right) \ldots f\left(s_{m+n}\right)\right\}$ \\
\hline
\end{tabular}

\section{Conclusion}

Web services provide the means for such seamless integration of business processes across organizational boundaries. How to integrate the existent Web services to form a newly value-added and complex service to meet the requirement of different users is a popular research area. This paper discusses the composition of Web Services using QoS. The idea is based on the assumption that for each task in a workflow a set of alternative Web Services with similar functionality is available and that these Web Services have different QoS parameters and costs. Web Service selection with QoS global optimal is transformed into a multi-objective services composition optimization with QoS constraints.

\section{Acknowledgement}

A Project Supported by Scientific Research Fund of Zhejiang Provincial Education Department (Grant No. Y201328355).

\section{References}

[1] Gong S, Wang Y. Research on Security Strategy of Electronic Commerce Industry Websites, 2015 International Conference on Advances in Mechanical Engineering and Industrial Informatics. Atlantis Press, 2015.

[2] Gong S. Research on the Generated Path of Electronic Commerce System Based on Innovative High-skilled Technology, 2015 International Industrial Informatics and Computer Engineering Conference. Atlantis Press, 2015.

[3] Songjie Gong, Liping Zeng, The Solution of Safety of Electronic Cash in E-Commerce under Cloud Computing Environment, Advanced Materials Research, Vol. 989, pp: 4314-4317, 2014.

[4] ChenZhou,Liang-Tien Chia and $\mathrm{Bu}$-Sung Lee.DAML-QoS Ontology for WebServices[J] IT Professional,2006 7(3):33-41

[5] Sycara K,Widoff S,Larks K M.dynamic match-making among heterogeneous software agents in cyberspace[J].Autonomous Agents and MultiAgent Systems,2002,5(2):173-203

[6] Paolueci M,Kawamura T. Matching of Web services capabilities[A].In Web Conference (ISWC)[C],Springer-Ver-lag,2002:333-347

[7] Songjie Gong, Research on the Growth Mechanism of High-Skilled System in Computer Science and Technology, Applied Mechanics and Materials, Vol. 513, pp: 2748-2751, 2014. 